Editorial

\title{
Acknowledgement to Reviewers of Coatings in 2014
}

Coatings Editorial Office, MDPI AG, Klybeckstrasse 64, CH-4057 Basel, Switzerland

Published: 8 January 2015

The editors of Coatings would like to express their sincere gratitude to the following reviewers for assessing manuscripts in 2014:

\begin{tabular}{lll} 
Abdul-Aziz, Ali & Colaco, Rogerio & He, Zhuliang \\
Agüero, Alina & Dang, Minh Trung & Ho, Wen-Fu \\
Ahmed, Mukhtar H. & Daviðsdóttir, Svava & Hohn, Keith \\
Akolkar, Rohan & Delabouglise, Didier & Horiuchi, Yu \\
Ali, El-Araby & Di Bella, G. & Hsiao, Kuang-Che \\
All, Angelo Homayoun & Dibb, George & Huang, Jung-Jie \\
Almtoft, Klaus Pagh & Duan, Fei & Ilavsky, Jan \\
Amadelli, R. & Edalati, Kaveh & Innocenti, Massimo \\
Ando, Yasutaka & Ellingson, Randy J. & Ishizuka, Shogo \\
Anfosso-Lédée, Fabienne & El-Mahallawy, Nahed & Ivanova, Elena P. \\
Bahamonde, A. & Evans, Drew & Iwasaki, Tomohiro \\
Baran, Derya & Faber, Hendrik & Jackson, R. W. \\
Barker, Philip J. & Fauchais, Pierre & Jorge-Sobrido, Ana \\
Beake, Ben & Fernandes, F. & Juarez, Manuel C. \\
Berardi, Umberto & Forsyth, Maria & Kandola, Baljinder \\
Birzer, C. H. & Foster, David G. & Kern, Wolfgang \\
Blayo, Anne & Fuentes, Gonzalo Garcia & Kim, Choong-Un \\
Bondioli, Federica & Fukuda, Takeshi & Kim, Dae Eun \\
Bonetta, Silvia & Georgiou, E. P. & King, Peter C. \\
Bosio, Alessio & Girard-Lauriault, Pierre-Luc & Kingshott, Peter \\
Cai, Zhongyu & Goforth, Andrea M. & Krysinski, Pawel \\
Cesbron, Julien & Guillén, C. & Krzanowski, James E. \\
Chae, Heeyeop & Gun'ko, Yurii & Lantieri, Claudio \\
Chen, Po-Yu & Gupta, Surojit & Lavacchi, Alessandro \\
Chrigui, M. & Hammond, Scott R. & Li, K. Y. \\
Coclite, Anna Maria & Hanaor, Dorian & Li, Sunny R. \\
& & \\
\hline & &
\end{tabular}


Lipkowski, Jacek

Lira-Cantu, Monica

Liu, Min

Liu, Yingchi

Lu, Qing

Mäkelä, Jyrki M.

Malucelli, Giulio

Mändl, Stephan

Martins, Rodrigo

Masami, Ikeyama

Masuda, Yoshitake

Mauer, Georg

Mishra, Yogendra

Mosquera, Maria

Murdoch, B. J.

Navarro-Martinez, S.

Paje, S. E.

Palmisano, Leonardo

Panagopoulos, C. N.

Pang, Y. X.
Parnell, Andrew J.

Perrenoud, Julian

Persson, Nils-krister

Plana, Daniela

Praticò, Filippo Giammaria

$\mathrm{Pu}$, Ying-Chih

Radenberg, Martin

Ravelli, Davide

Reverdy-Bruas, Nadege

Rizzello, Loris

Rosa, Roberto

Röttger, A.

Sandberg, Ulf

Sarfraz, Adnan

Schilardi, P. L.

Shimizu, Kazuo

Silva, F. J. G.

Soebarto, Veronica

Song, Yi

Steinmetz, Nicole
Suresh, K.

Sykes, John

Tait, Jeffrey G.

Tsay, Chien-Yie

Van de Ven, Martin

Vasiljevic, Natasa

Ward, L. P.

Weber, Miriam

Wise Sr., John Pierce

Wynne, Kenneth

Xue, Wei

Yamaguchi, Seiji

Ye, Qiaoyan

Yoshino, Kenji

Yoshioka, Yasuo

Yu, Jing

Zangari, Giovanni

Zhao, Q.

Zhuravleva, Ksenia

(C) 2015 by the authors; licensee MDPI, Basel, Switzerland. This article is an open access article distributed under the terms and conditions of the Creative Commons Attribution license (http://creativecommons.org/licenses/by/4.0/). 